\title{
SEMI-LOCAL-CONNECTEDNESS AND CUT POINTS IN METRIC CONTINUA
}

\author{
E. D. SHIRLEY
}

\begin{abstract}
In the first section of this paper, the notion of a space being rational at a point is generalized to what is here called quasi-rational at a point. It is shown that a compact metric continuum which is quasi-rational at each point of a dense subset of an open set is both connected im kleinen and semi-locally-connected on a dense subset of that open set. In the second section a $G_{\delta}$ set is constructed such that every point in the $G_{\delta}$ at which the space is not semi-locally-connected is a cut point. A condition is given for this $G_{\delta}$ set to be dense. This condition, in addition to requiring that the space be not semi-locally-connected at any point of a dense $G_{\delta}$ set gives a sufficient condition for the space to contain a $G_{\delta}$ set of cut points. The condition generalizes that given by Grace.
\end{abstract}

1. Throughout this paper $M$ will be taken to be a compact metric continuum. Many of the lemmas, however, can be proven with less hypotheses. Lemma 2, for example, requires only that the sets $P_{x}$ (defined below) be subcontinua of $M$. Compact Hausdorff is sufficient for this to happen [4].

Let $x, y$, and $z$ be points of $M$ (not necessarily distinct). The point $x$ cuts between $y$ and $z$ in $M$ when every subcontinuum of $M$ which contains both $y$ and $z$ must also contain $x$. The point $x$ is a cut point of $M$ when $x$ cuts between two points distinct from $x . M$ is said to be aposyndetic (semi-locally-connected) at $x$ with respect to $y$ if and only if there is a subcontinuum of $M$ with $x(y)$ in its interior that does not contain $y(x)$. $M$ is aposyndetic (semi-locally-connected) at $x$ when it is aposyndetic (semi-locally-connected) at $x$ with respect to every other point. Finally, $M$ is connected im kleinen at $x$ when each neighborhood of $x$ contains a closed neighborhood of $x$ which is also connected. One should note that when $M$ is connected im kleinen at a point, it is also aposyndetic at that point. For $x \in M, P_{x}$ denotes $\{y \in M \mid M$ is not aposyndetic at $y$ with respect to $x\}$, and for $T \subseteq M, P_{T}^{\prime}$ denotes $\bigcap\left\{H \mid T \subseteq H^{0}\right.$ and $H$ is a

Received by the editors May 20, 1970.

AMS 1969 subject classifications. Primary 5455.

Key words and phrases. Aposyndetic, semi-locally-connected, connected im kleinen, quasi-rational curve, cut point, $G_{\delta}$ set of cut points.

(c) American Mathematical Society 1972 
subcontinuum of $M$ \}. Clearly $M$ is semi-locally-connected at $x$ if and only if $P_{x}=\{x\}$. As noted above, $P_{x}$ is a subcontinuum of $M$.

LEMMA 1.1. If $T$ is a subcontinuum of $M$ and $x$ is a point of $M$, then $x \in P_{T}^{\prime}$ if and only if $P_{x} \cap T \neq \varnothing$.

Proof. Suppose $x \in P_{T}^{\prime}$. If $P_{x} \cap T=\varnothing$, then $M$ is aposyndetic at every point of $T$ with respect to $x$. By the definition of aposyndetic and the compactness of $T$ we see that $T$ can be covered by the interior of a finite number of continua $A_{1}, \cdots, A_{n}$, where each $A_{i}$ meets $T$ and does not contain $x$. Now $T \cup \cup_{i=1}^{n} A_{i}$ is a subcontinuum of $M$ containing $T$ in its interior, and hence $x \in P_{T}^{\prime} \subseteq T \cup \cup_{i=1}^{n} A_{i}$. Since $x \notin A_{i}$, we have $x \in T$, and so $x \in P_{x} \cap T=\varnothing$. This contradiction shows $P_{x} \cap T \neq \varnothing$.

Conversely, suppose that for some $x \in M, P_{x} \cap T \neq \varnothing$, say $y \in P_{x} \cap T$. Let $T \subseteq H^{0}$ where $H$ is a subcontinuum of $M$. Then $y \in H^{0}$. Since $y \in P_{x}$, $M$ is not aposyndetic at $y$ with respect to $x$. It follows that $x \in H$. Thus $x \in P_{T}^{\prime}$.

LEMMA 1.2. If $T$ is a subcontinuum of $M$, then for $z \in\left(P_{T}^{\prime}\right)^{0}$ and $y \in$ $M-T$ we have $z \in P_{y}$ implies $y \in P_{z}$.

Proof. Suppose $y \notin P_{z}$ and $z \in P_{y}$. Then there is a continuum $H$ containing $y$ in its interior which does not contain $z$. Let $U$ be an open neighborhood of $y$ in $H^{0} \cap(M-T)$, and let $L$ be the component of $M-U$ containing $T$. Suppose $x \in(M-H) \cap P_{T}^{\prime}$. Then in particular $P_{x} \cap T \neq \varnothing$. If $x \notin L$, then $L$ is a proper subset of $P_{x} \cup L$. Hence $P_{x} \cap U \neq \varnothing$. Let $s \in P_{x} \cup U$, then $s \in H^{0}$ and thus $x \in H$. With this contradiction we conclude that $(M-H) \cap P_{T}^{\prime} \subseteq L$. Thus $z \in(M-H) \cap$ $\left(P_{T}^{\prime}\right)^{0} \subseteq L^{0}$ and, of course, $z \in P_{y}$. This implies $y \in L$ which contradicts the fact that $y \in U \subseteq M-L$.

LEMMA 1.3. Let $V$ be an open point set of $M$. M is semi-locally-connected on a dense subset of $V$ if and only if for each open point set $W$ in $V$, there is a finite number of continua covering $\partial W$ but not all of $W$.

Proof. That this condition is necessary is immediate, for if $W$ is an open point set of $V$, then there is a point $x$ in $W$ at which $M$ is semilocally-connected. Thus $M$ is aposyndetic at each point of $\partial W$ with respect to $x$. Since $\partial W$ is compact we can conclude there is a finite number of continua covering $\partial W$ with their interior but not containing $x$.

Conversely let $W$ be any open point set of $V$. We will find a point $x \in W$ at which $M$ is semi-locally-connected. By the hypothesis we can 
choose open point sets $W_{i}$ and continua $H_{1}^{i}, \cdots, H_{n_{i}}^{i}$ such that

(1) $W_{1} \subseteq W$,

(2) $\partial W_{i} \subseteq \bigcup_{j=1}^{n_{i}} \cdot H_{j}^{i}$,

(3) $\bar{W}_{i+1} \subseteq W_{i}-\bigcup_{j=1}^{n_{i}} H_{j}^{i}$,

(4) $x, y \in W_{i}$ implies $d(x, y) \leqq 1 / i$.

Let $x \in \bigcap W_{i}$. For $y \neq x$, choose $k$ such that $y \notin N_{2 / k}(x)\left(N_{r}(x)\right.$ is the open ball with center $x$ and radius $r)$. Then $x \in\left(W_{k}-\bigcup_{j=1}^{n_{k}} H_{j}^{k}\right)=U$, and $y \in M-\bar{U}$. Now each component of $M-\bar{U}$ meets $\partial U$ which is in $\bigcup_{j=1}^{n_{k}} H_{j}^{k}$. Thus $M-U$ has only a finite number of components (each component of $M-U$ contains at least one $H_{j}^{k}$. Since $y \in M-\bar{U}, y$ is in the interior of the component of $M-U$ containing $y$. Since this component does not contain $x, M$ is semi-locally-connected at $x$ with respect to $y$. It follows that $M$ is semi-locally-connected at $x$ which completes the proof.

$M$ is said to be quasi-rational at $x$ if and only if for each open neighborhood $W$ of $x$ there is an open neighborhood $U$ of $x$ in $W$ such that $W-U$ contains a closed set which is a countable union of continua and which separates $U$ from $M-W$.

LEMMA 1.4. If $M$ is quasi-rational on a dense subset of an open point set $V$ of $M$, then $M$ is connected im kleinen on a dense subset of $V$.

Proof. Let $W$ be an open point set in $V$. We will show $W$ contains a point at which $M$ is connected im kleinen. $W$ contains a point at which $M$ is quasi-rational. Thus there is an open point set $U$ and continua $T_{1}, T_{2}, \cdots$ such that $U T_{i} \subseteq W-U$ is closed and separates $U$ from $M-W$. Since each component of $M-\cup T_{i}$ meets some $T_{i}$, we see $U$ is covered by a countable number of continua in $W$. One of these continua must contain an open subset of $U$. The above proof procedure allows us to verify that there are continua $H_{1}, H_{2}, \cdots$ in $W$ such that for each positive integer $i, H_{i+1} \subseteq H_{i}^{0}$ and the diameter of $H_{i}$ is $\leqq 1 / i$. Let $x \in \bigcap H_{i}$. Then since $x \in H_{i}^{0}$ for each $i$ and for each neighborhood $G$ of $x$ there exists an integer $i$ such that $H_{i}$ is contained in $G, M$ is connected im kleinen at $x$.

THEOREM 1.1. If $M$ is quasi-rational on a dense subset of an open point set $V$ then $M$ is semi-locally-connected on a dense subset of $V$.

Proof. Suppose not. By Lemma 1.3 there is an open point set $W$ in $V$ such that if $\partial W$ is covered by a finite number of continua, then they cover all of $W$. This implies in particular that $P_{x} \cap \partial W \neq \varnothing$ for all $x \in W$. Now let $U$ be an open point set in $W$ which is separated from $M-W$ by a countable union of continua, $\bigcup T_{i}$, which is a closed subset of $W-U$. Since $P_{x}$ is connected and $P_{x} \cap \partial W \neq \varnothing$ for $x \in W$, for $x \in U$ we have $P_{x} \cap \bigcup T_{i} \neq \varnothing$. Let $K_{i}=\left\{x \in U \mid P_{x} \cap T_{i} \neq \varnothing\right\}$. 
It is easily seen that $K_{i}$ is a closed subset of $U$ (relative topology) for each $i$ [4, Theorem 1]. Since the $K_{i}$ 's form a countable cover of $U$, it follows that for some $i, K_{i}^{0} \neq \varnothing$. By Lemma 1.1 we see $K_{i}^{0} \subseteq P_{T_{i}}^{\prime} \cap$ $\left(M-T_{i}\right)$. By Lemma 1.4 there is a point $x \in U \cap\left(P_{T_{i}}^{\prime}\right)^{0}$ at which $M$ is aposyndetic. Since $P_{x} \cap \partial W \neq \varnothing$ and $P_{x}$ is connected, there is a point $y \in U \cap\left(P_{T_{i}}^{\prime}\right)^{0} \cap P_{x}$ different from $x$. By Lemma 1.2, $x \in P_{y}$ which contradicts the fact that $M$ is aposyndetic at $x$ with respect to $y$.

2. In the following a $G_{\delta}$ set is constructed such that every point in the $G_{\delta}$ at which $M$ is not semi-locally-connected is a cut point. Then it is proven that under certain conditions this $G_{\delta}$ is dense. In this section $y$ is a fixed point of $M . C(x, i)$ is used to denote the component of $M-N_{1 / i}(x)$ containing $y$, and when it is used it is assumed that $y \in$ $M-N_{1 / i}(x)$. Let $G_{n}=\{z \in M \mid$ there is a point $x \in M$ and integers $i, j$ such that $d(x, z)<1 / n, i>n$, and $\left.C(x, i) \subseteq C(z, j)^{0}\right\}$ and let $G=\bigcap G_{n}$.

LEMMA 2.1. $G_{n}$ is an open set for each $n$.

Proof. Let $z \in G_{n}$. There is a point $x$ of $M$ and integers $i, j$ such that $d(x, z)<1 / n$, and $C(x, i) \subseteq C(z, j)^{0} . N_{1 / n}(x) \cap N_{1 / j}(z)$ is a neighborhood of $z$. For $s \in N_{1 / n}(x) \cap N_{i / j}(z)$ we have $d(x, s)<1 / n$, and we can find a $k$ so that $N_{1 / k}(s) \subseteq N_{1 / j}(z)$. Hence $C(z, j) \subseteq C(s, k)$. It follows that $C(s, i) \subseteq C(z, j)^{0} \subseteq C(s, k)^{0}$, and thus $s \in G_{n}$.

Lemma 2.2. If $z \in G$ and $z$ is not a cut point, then $M$ is semi-locallyconnected at $z$.

Proof. Suppose $z \in G$ is not a cut point. For each positive integer $n$ there exists a point $x_{n}$ and integers $i_{n}, j_{n}$ such that $d\left(x_{n}, z\right)<1 / n, i_{n}>n$ and $C\left(x_{n}, i_{n}\right) \subseteq C\left(z, j_{n}\right)^{0}$. Now $C\left(z, j_{n}\right)$ is a continuum not containing $z$, so $P_{z} \subseteq \bigcap_{n}\left(M-C\left(z, j_{n}\right)\right)^{0} \subseteq \bigcap_{n}\left(M-C\left(x_{n_{j}}, i_{n}\right)\right)$. Suppose $s \in$ $\bigcap_{n}\left(M-C\left(x_{n}, i_{n}\right)\right)$ and $s \neq z$. Let $H$ be a subcontinuum of $M$ joining $s$ to $y$ and missing $z$. Choose $k$ large enough so that $N_{1 / k}(z) \cap H=\varnothing$. Then $H \subseteq C(z, k)$. Also choose $p$ large enough so that $N_{1 / i_{p}}\left(x_{p}\right) \subseteq$ $N_{1 / k}(z)$. Then $C(z, k) \subseteq C\left(x_{p}, i_{p}\right)$ and hence $s \in C\left(x_{p}, i_{p}\right)$. This contradiction shows $\bigcap\left(M-C\left(X_{n}, i_{n}\right)\right) \subseteq\{z\}$. Thus $P_{x} \subseteq\{z\}$ and $M$ is semilocally-connected at $z$.

THEOREM 2.1. Let $V$ be an open set in $M$. Suppose for all continua $T$ containing $y$ we have that $\left(P_{T}^{\prime}\right)^{0} \cap(V-T)=\varnothing$, then $V \cap G$ is dense in $V$.

Proof. Suppose $W \subseteq V-G$ is an open point set of $M$. Let $x_{1} \in W$. Choose $i_{1}>1$ such that $N_{1 / i_{1}}\left(x_{1}\right) \subseteq W$ (with no loss of generality $y \notin W$ ). If there is an $x \in N_{1 / i_{1}}\left(x_{1}\right)$ such that, for some $j, C\left(x_{1}, i_{1}\right) \subseteq C(x, j)^{0}$ then 
we let $x_{2}=x$ and $i_{2}=\max (j, 2, k)$ where $k$ is such that

$$
\overline{N_{1 / k}(x)} \subseteq N_{1 / i_{1}}\left(x_{1}\right)
$$

Suppose for each positive integer $n$ there exists a point $x_{n}$ and an integer $i_{n}>n$ such that

$$
C\left(x_{n}, i_{n}\right) \subseteq C\left(x_{n+1}, i_{n+1}\right)^{0} \text { and } \overline{N_{1 / i_{n+1}}\left(x_{n+1}\right)} \subseteq N_{1 / i_{n}}\left(x_{n}\right) .
$$

Let $z \in \bigcap N_{1 / i_{n}}\left(x_{n}\right)$. Then $x_{1}, x_{2}, x_{3}, \cdots$ converges to $z$. Since $z \in$ $N_{1 / i_{n+1}}\left(x_{n+1}\right)$, for each positive integer $n$ there is a $j_{n}$ such that $N_{1 / j_{n}}(z) \subseteq$ $N_{1 / i_{n+1}}\left(x_{n+1}\right)$. Hence we conclude that $C\left(x_{n}, i_{n}\right) \subseteq C\left(x_{n+1}, i_{n+1}\right)^{0} \subseteq$ $C\left(z, j_{n}\right)^{0}$. Since $d\left(x_{n}, z\right)<1 / n$ it follows that $z \in G_{n}$ for all $n$. But this says $z \in G \cap W$. We conclude that there must be an $n$ such that $x \in$ $N_{1 / i_{n}}\left(x_{n}\right)$ implies $C\left(x_{n}, i_{n}\right) \nsubseteq C(x, j)^{0}$ for all $j$. Let $T=C\left(x_{n}, i_{n}\right) . T$ is a subcontinuum of $M$ containing $y$. Let $s \in N_{1 / i_{n}}\left(x_{n}\right)$ and $T \subseteq H^{0}$ where $H$ is a subcontinuum of $M$. If $s \notin H$, then there is a $j$ such that $N_{1 / j}(s) \subseteq$ $M-H$. Hence $H \subseteq C(s, j)$. This says $C\left(x_{n}, i_{n}\right)=T \subseteq H^{0} \subseteq C(s, j)^{0}$ which is a contradiction. Therefore $N_{1 / i_{n}}\left(x_{n}\right) \subseteq H$. It follows that $N_{1 / i_{n}}\left(x_{n}\right) \subseteq P_{T}^{\prime} \cap(V-T)$, contradicting the fact that

$$
\left(P_{T}\right)^{0} \cap(V-T)=\varnothing \text {. }
$$

Corollary 2.1. If $M$ is not semi-locally-connected at any point of a dense $G_{\delta}$ subset of an open point set $V$, and if for any subcontinuum $T$ of $M$ containing $y$ we have $\left(P_{T}^{\prime}\right)^{0} \cap(V-T)=\varnothing$. Then $V$ contains a dense $G_{\delta}$ set of cut points.

Corollary 2.2 (Grace [2]). Suppose $V$ is an open set of $M$ which contains a dense $G_{\delta}$ set $G$ such that given any point $x$ in $G, M$ is locally peripherally aposyndetic at $x$ and $M$ is not semi-locally-connected at $x$. Then $V$ contains a dense $G_{\delta}$ set of cut points.

( $M$ is locally peripherally aposyndetic at $x$ when for $x \in U, U$ open, there is an open set $W$ such that $x \in W \subseteq U$ and $M$ is aposyndetic at $x$ with respect to each point of $\partial W$.)

Proof. If $V$ does not contain a dense $G_{\delta}$ set of cut points, then by Corollary 2.1 there is a continuum $T$ such that $\left(P_{T}^{\prime}\right)^{0} \cap(V-T) \neq \varnothing$. Let $x \in\left(P_{T}^{\prime}\right)^{0} \cap(V-T)$ be a point at which $M$ is both locally peripherally aposyndetic and not semi-locally-connected. Since $M$ is not semi-locallyconnected at $x$, there is an open set $W$ such that $x \in W \subseteq\left(P_{T}^{\prime}\right)^{0} \cap(V-T)$ and $P_{x} \cap(M-W) \neq \varnothing$. Let $U$ be open such that $x \in U \subseteq W$ and $M$ is aposyndetic at $x$ with respect to each point of $\partial U$. Since $P_{x}$ is a continuum there is a $z \in P_{x} \cap \partial U$. By Lemma 1.2, $x \in P_{z}$. But this says $M$ is not aposyndetic at $x$ with respect to $z$ and since $z \in \partial U$ we have a contradiction. 
Jones [4, Theorem 15] has shown that a compact metric continuum $M$ which is not semi-locally-connected at any of its points contains a dense set of cut points. Grace [1] posed the question whether a space $M$ has a $G_{\delta}$ set of cut points. In particular, this would imply that the cardinality of the collection of cut points is $c$. Hagopian [3, Theorem 4] has shown that the latter must happen: If a compact metric continuum $M$ is not semilocally-connected at any point of a $G_{\delta}$ subset which is dense in $M$ then the set of cut points in each open point set has cardinality $c$.

Suppose $V$ is open in $M$ and $M$ is not semi-locally-connected at any point of a dense $G_{\delta}$ subset $K$ of $V$. Let $V_{1}=V \cap\left(\bigcup\left\{P_{T}^{\prime 0}-T \mid T\right.\right.$ is a subcontinuum of $M\})$ and let $V_{2}=\left(V-V_{1}\right)^{0}$. By Corollary 2.1, $V_{2}$ contains a dense $G_{\delta}$ set of cut points. Although we cannot show that $V_{1}$ contains a dense $G_{\delta}$ set of cut points (which would answer Grace's question), we can strengthen Hagopian's result by proving that when $V_{1} \neq \varnothing, V_{1}$ contains a nondegenerate continuum whose points are cut points. Assume $V_{1} \neq \varnothing$.

THEOREM 2.2. $V_{1}$ contains a dense $G_{\delta}$ set $J$ such that for each $x \in J$ there is a nondegenerate subcontinuum $H$ of $M$ containing $x$ such that each point of $H$ cuts $x$ from $y$.

Proof. As a special case of Grace's Theorem 2 [2] we have $M$ contains a dense $G_{\delta}$ set $I$ such that if $x \in I \cap P_{z}$ then $z$ cuts $x$ from $y$. Let $J=V_{1} \cap I$. For $x \in J$ there is a subcontinuum $T$ of $M$ such that $x \in$ $P_{T}^{\prime 0}-T$. Let $H$ be any nondegenerate subcontinuum of $P_{x}$ in $P_{T}^{\prime 0}$ containing $x$. By Lemma 1.2, $z \in H$ implies $x \in P_{z}$. Since $x \in I, z$ cuts $x$ from $y$.

By choosing a nondegenerate subcontinuum $K$ of $H$ (the $H$ of Theorem 2.2) which is contained in $V-\{x, y\}$, we have that each point of $K$ is a cut point.

\section{REFERENCES}

1. E. E. Grace, Cut sets in totally nonaposyndetic continua, Proc. Amer. Math. Soc. 9 (1958), 98-104. MR 20 \#1960.

2. - Cut points in totally non-semi-locally-connected continua, Pacific J. Math. 14 (1964), 1241-1244. MR 30 \#4243.

3. C. L. Hagopian, On generalized forms of aposyndesis, Pacific J. Math. 34 (1970), 97-108.

4. F. B. Jones, Concerning non-aposyndetic continua, Amer. J. Math. 70 (1948), 403-413. MR 9 \#606.

Department of Mathematics, University of Illinois, Urbana, Illinois 61801 Current address: Bethune-Cookman College, Daytona Beach, Florida 32015 